\title{
Nuanced roles of cytokines in three major human brain disorders
}

\author{
Lawrence Steinman \\ Department of Neurology and Neurological Sciences, Interdepartmental Program in Immunology, \\ Stanford University School of Medicine, Stanford, California, USA.
}

\begin{abstract}
The three most prevalent human disorders of the CNS in which immunity and inflammation are likely to have vital roles (excluding infection of the CNS) are fever, multiple sclerosis (MS), and Alzheimer disease (AD). As reviewed here, cytokines are critical in the induction of fever, the pathogenesis of MS, and the pathobiology of AD. Indeed, antibodies targeting cytokines have been used as a therapy for individuals with unusual and persistent febrile reactions not responsive to common antipyretics, while a recombinant cytokine is the most popular treatment for the relapsing-remitting form of MS. Although cytokine-modulating therapies are not currently in clinical use for the treatment of $\mathrm{AD}$, cytokines can ameliorate disease pathology in certain experimental models of $\mathrm{AD}$, suggesting a potential for future therapeutic opportunities.
\end{abstract}

\section{Introduction}

The CNS was considered for many years to be an immune-privileged site - a region of the body that does not mount a normal inflammatory immune response to foreign antigen. However, it is now clear that inflammatory immune responses can occur in the CNS. Although these responses differ from classical inflammatory immune responses in the periphery, many of the same cells are involved. For example, immune responses in the CNS involve not only activation of resident cells (including microglial cells, astrocytes, and endothelial cells), but also infiltration of circulating immune cells (including monocytes, neutrophils, and $\mathrm{T}$ cells). As in the periphery, both the activated resident cells and the infiltrating cells express, release, and/or respond to proand antiinflammatory cytokines, which function in the CNS as both regulators of immunity and modulators of neuronal and glial function. Of particular interest when considering pathologic inflammatory conditions in the CNS, many cells of the glial lineage secrete proinflammatory cytokines such as IFN- $\gamma$ and TNF- $\alpha$ (1). A second similarity to the periphery that is important to bear in mind is that different inflammatory insults result in the induction of diverse inflammatory phenotypes, involving distinct cells and particular cytokine mediators. Consistent with this, the study of cytokine expression and action in multiple models of pathologic inflammatory conditions in the CNS has revealed that cytokines play diverse roles, at times providing signals for inflammation, while at other times providing signals for neuroprotection, inducing, in a Janus-like manner, neurological disease and then brain repair (1). The nuanced, intricate, double-edged role of cytokines in pathologic inflammatory conditions in the CNS makes understanding the timing and context of their presence a matter of utmost importance.

This Review focuses on the role of cytokines in three of the most prevalent human disorders of the CNS in which immunity and inflammation play vital roles. Infectious diseases of the CNS, including parasitic infections and HIV-induced encephalopathy,

Nonstandard abbreviations used: $\mathrm{A} \beta$, amyloid $\beta ; \mathrm{AD}$, Alzheimer disease; $\mathrm{BBB}$, blood-brain barrier; IFNAR, type I IFN receptor; TF, tissue factor.

Conflict of interest: The author has declared that no conflict of interest exists.

Citation for this article: J. Clin. Invest. 118:3557-3563 (2008). doi:10.1172/JCI36532. have been intentionally excluded here so that the focus is on fever, MS, and Alzheimer disease (AD). While there have been many articles that review the role of cytokines in animal models of CNS diseases (e.g., EAE models of MS), this article only refers to such studies when they illuminate a principle in one of the three human conditions discussed. For example, if one is looking for a review of the roles of Th1 and Th17 in EAE, which have recently been under intense scrutiny, several comprehensive pieces are available (including refs. 2-5). Instead, I discuss what is known about the roles of these Th cell subsets in MS, which means that the discussion of the Th17 pathway is somewhat limited, because it has hardly been analyzed in MS itself as yet (5).

\section{Fever}

What is fever? Fever (also known as pyrexia and the febrile response) is a condition so common that all of us have experienced it numerous times in our lives. It is defined as a regulated increase in internal body temperature to levels above normal (in adults, an oral temperature greater than $99.5^{\circ} \mathrm{F}$ is typically considered to constitute a fever), usually in response to infection, and is triggered by stimulation of thermoregulatory neurons in the hypothalamus at the base of the brain.

The role of cytokines in fever. The history of the discovery of cytokines is inextricably entwined with investigations into the triggers for the febrile response - so much so that two years before the laboratories of Charles Dinarello and Steven Mizel independently cloned IL-1 and found that it was the same as the fever-inducing factor produced by leukocytes and known as leukocyte pyrogen (6-8), Dinarello had outlined in a review article the mechanism by which he thought leukocyte pyrogen was likely to initiate fever. He also predicted the likely mechanism whereby antipyretics prevent the synthesis of COX metabolites and therefore reduce fever (9). These proposed mechanisms still hold true today, explaining the roles of IL- 1 in the genesis of fever and how antipyretics work to reduce fever.

Thus, there is a wonderful congruence in the history of molecular immunology that the primary and perhaps quintessential manifestation of inflammation and fever is mediated by the very first cytokine to be cloned (IL-1). Indeed, as Dinarello hypothesized for leukocyte pyrogen (9), IL-1 alerts the hypothalamus that there is "danger" in the periphery (1), as do other pyrogenic 


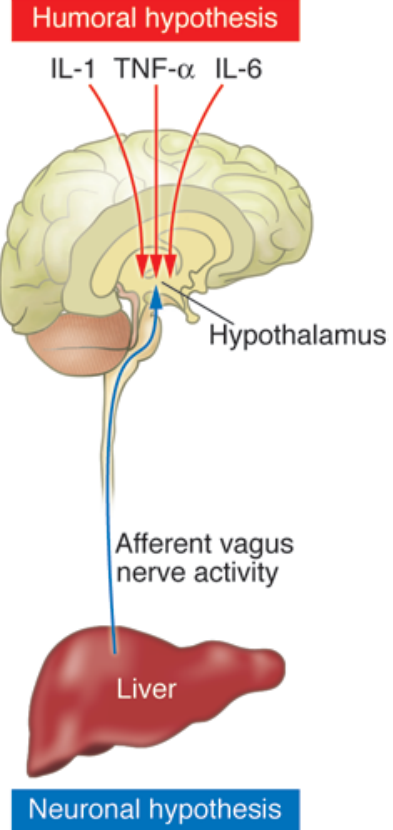

Figure 1

Comparison of the humoral and neuronal hypotheses for fever induction. According to the humoral hypothesis of fever induction, the pyrogenic cytokines IL-1, IL-6, and TNF- $\alpha$ gain access to the hypothalamus via fenestrations in the BBB in the circumventricular organs or via active transport mechanisms mediated by the cells surrounding the hypothalamus. A key feature of the humoral hypothesis is that the pyrogenic cytokines activate the febrile response indirectly, by inducing local endothelial cells or microglial cells to secrete $P G_{2}$. The $P G_{2}$ then acts via prostaglandin $E$ receptor 3 to initiate a neuronal response that regulates the body temperature. According to the neuronal hypothesis, C5a stimulates $\mathrm{PGE}_{2}$ production in the liver, and signaling to the hypothalamus occurs via a neural pathway mediated by the vagus nerve and the nucleus tractus solitarius.

signals, such as TNF- $\alpha$ and IL-6 (1). There is strong evidence of a humoral route of transmission for these pyrogenic cytokines from the periphery to the thermoregulatory neurons in the hypothalamus (Figure 1). According to this humoral hypothesis, pyrogenic cytokines such as IL-1, IL-6, and TNF- $\alpha$ activate the febrile response indirectly, by inducing the secretion of $\mathrm{PGE}_{2}$. Exactly where the $\mathrm{PGE}_{2}$ is produced and where it then goes to trigger neurons is a subject of intense research.

$\mathrm{PGE}_{2}$ acts upon local endothelial cells or closely situated glial cells just inside the brain in the region surrounding the brain's ventricular system. These cells in turn activate neurons in the preoptic area of the anterior hypothalamus $(10,11)$. The cells in this region of the brain surround portals in the blood-brain barrier (BBB) and are collectively known as the circumventricular organs. In these circumventricular organs, highly specialized fenestrations in the $\mathrm{BBB}$ allow transmission of cytokines to precise sites located in the hypothalamus of the brain. Signaling is via cytokine receptors for IL-1, TNF- $\alpha$, and IL- 6 on microglia cells. These receptors then activate the arachidonic acid pathway, causing release of $\mathrm{PGE}_{2}$. The $\mathrm{PGE}_{2}$ then acts on neurons in the preoptic area of the hypothalamus, which receive the signal via prostaglandin $E$ receptor 3 and respond by regulating temperature (11). Other researchers have provided evidence that there are active transport mechanisms for moving cytokines such as IL- 1 , TNF- $\alpha$, and IL- 6 across the BBB (12). These transport mechanisms may augment the effect of these pyrogenic cytokines by providing yet another route for them to access the hypothalamic centers (12).

These schemes are useful for explaining how fever arises after exposure to IL-1 and how salicylates (such as aspirin) and other antiinflammatory drugs might act to block prostaglandins and thus inhibit fever (11). The neurons in the preoptic area of the hypothalamus that are at the culmination of the cytokine/PGE 2 cascade are also connected with neurons that regulate sleep and eating. This helps explain, in part, why fever is associated with somnolence and loss of appetite.

Recently, Bartfai and colleagues have refined the humoral hypothesis for fever generation (11). There is a very rapid rise in core body temperature that occurs prior to the generation of $\mathrm{PGE}_{2}$, which requires NF- $\mathrm{KB}$ induction of transcription of $\mathrm{COX} 2$, a process that takes around 30 minutes (11). They showed that IL-1 $\beta$ mediated induction of sphingomyelinase via the receptor for IL-1 mediates a rapid febrile response that circumvents the need for $\mathrm{PGE}_{2}$ (11). Ceramide produced by the sphingomyelinase acts as the second messenger in place of $\mathrm{PGE}_{2}$ and triggers the rapid activation of preoptic neurons in the hypothalamus. Thus, the initial rapid rise in core body temperature is mediated via ceramide, and only after about 30-45 minutes, during which transcription of COX2 is activated, does the $\mathrm{PGE}_{2}$ pathway become responsible for the febrile response (10). Both the $\mathrm{PGE}_{2}$ pathway and the sphingomyelinase pathway are triggered via IL- $1 \beta$ and are orchestrated in the rapid and prolonged response manifest as fever.

There is also a second hypothesis to explain how fever is generated. As fever can occur independently of IL-1, IL-6, and TNF- $\alpha$, the alternative scheme is known as the neuronal hypothesis (13). According to the neuronal hypothesis, stimulation of Kupffer cells in the liver via LPS can signal a cascade of events mediated by complement component C5a $(14,15)$. C5a stimulates $\mathrm{PGE}_{2}$ production in the liver, and signaling to the hypothalamus occurs via a neural pathway mediated by the vagus nerve and the nucleus tractus solitarius. The neuroanatomical circuit projects to the ventromedial preoptic nerves in the hypothalamus. Such a pathway triggers febrile responses and may act independently or in concert with the humoral pathways discussed above (13).

Whether one or the other hypothesis is correct or whether both merge together to explain the physiology of fever needs to be resolved with further research. What is clear, however, is that anticytokine therapy has been used successfully to block particularly vexing conditions involving fever. In some patients with various infectious diseases, including louse-borne relapsing fever, secondary syphilis, brucellosis, leptospirosis, and Lyme disease, treatment with antibiotics is followed by a rise in TNF- $\alpha$, IL-6, and IL-8 levels and then a devastating fever (16). This is known as the Jarisch-Herxheimer reaction, and it is thought to occur when large quantities of toxins are released into the body when the antibiotics kill the disease-causing bacteria. For example, individuals with louse-borne relapsing fever (which is caused by infection with Borrelia recurrentis), when treated with antibiotics, sometimes develop a devastating reaction manifest by fever, rigor, and hypotension. These violent reactions can be blocked with TNF- $\alpha$-specific antibodies (17). The role of IL-1, TNF- $\alpha$, and other cytokines in fever can certainly be dramatically demonstrated in this scenario. Amelioration of fever, rigor, and shock-like hypotension with cytokine-specific antibodies 


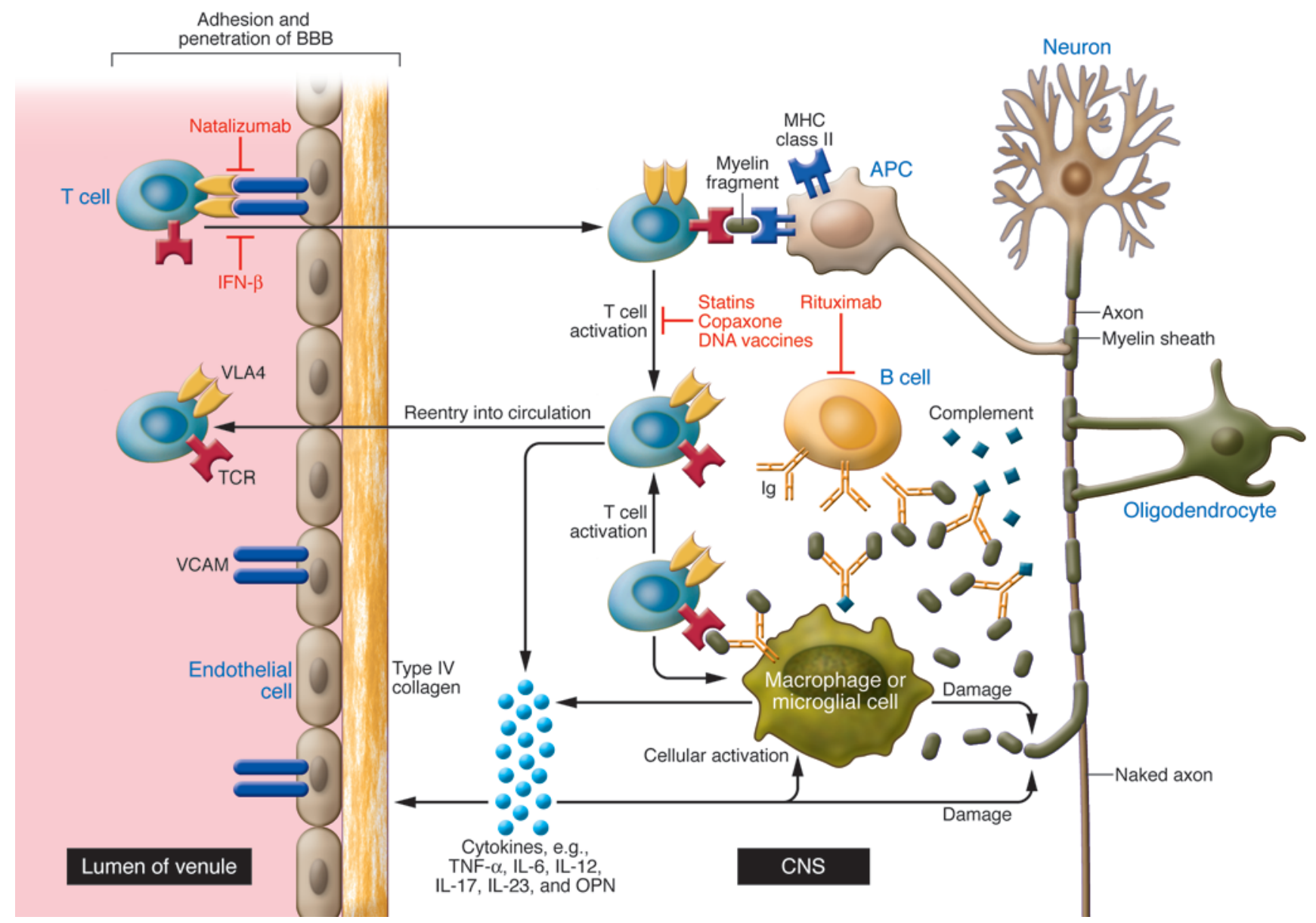

\section{Figure 2}

Many cytokines influence the pathogenesis of MS. MS is a neurodegenerative autoimmune disease of the white matter of the CNS in which cytokines have an important role. Some cytokines, such as IL-6, IL-17, TGF- $\beta$, and IFN- $\gamma$, trigger an inflammatory response in the white matter of the brain. Such inflammatory responses contribute to the development of plaques (the characteristic pathology of MS) in the white matter by stripping myelin from neurons. Other molecules that regulate cytokines (e.g., osteopontin [OPN]) may increase Th17 and IFN- $\gamma$ production. In addition, the most popular treatment for individuals with MS is the cytokine IFN- $\beta$, which is though to attenuate the action of the proinflammatory cytokines. Depicted are other potential therapies that may involve blockade of IFN- $\gamma$, IL-6, IL-12, or IL-23. Therapies aimed at blocking the adaptive immune response include DNA vaccination to induce tolerance. Statins and Copaxone are aimed at treatment of innate immune pathways and at the immune synapse. The most potent approved therapy for MS, natalizumab, blocks $\alpha_{4} \beta_{1}$ integrin (verly late antigen 4 [VLA4]) interactions with VCAM. Depletion of B cells with anti-CD20 may be a promising therapeutic approach (all these approaches are discussed in refs. 29, 63, and 64). Adapted with permission from Nature Reviews. Immunology (23).

demonstrates the importance of the role these cytokines play in the most severe clinical variants of the febrile response.

\section{MS}

What is MS? MS is a neurodegenerative autoimmune disease of the white matter of the CNS. It affects over one million people worldwide, women twice as frequently as men. Disease onset usually occurs in young adults as a relapsing-remitting disease, with recurrent attacks producing a range of neurological deficits, including blindness, loss of sensation, and lack of coordination. Symptoms can disappear completely between attacks but often persist as the disease advances.

Neurodegeneration in MS is a consequence of demyelination, which generates the plaques in the white matter that are the characteristic pathology of the disease. Specifically, a plaque is an area of white matter in which the inflammatory response has stripped myelin from the neurons, and then nonneuronal cells in the brain, including microglial cells, have caused scarring. The CNS in individuals with MS is filled with perivascular inflammatory cuffs containing lymphocytes and macrophages, a feature that is conspicuously absent in $\mathrm{AD}$ (see below). In the CNS of individuals with MS, the cells in these cuffs as well as the cells that exit from the cuffs to enter the brain provide all the cellular components necessary for the promulgation of an adaptive immune response within the brain.

Although the cause of MS has remained elusive, much is known about the pathogenesis of the disease, with many cytokines having a major impact (Figure 2). There are three well-established examples of the role of cytokines in MS: first, IFN- $\gamma$ administration exacerbates MS; second, the most popular treatment for MS is the cytokine IFN- $\beta$, which is mildly successful in reducing the relapse rate in patients with MS; and last, blockade of TNF- $\alpha$ is 
associated with worsening of MS. These will be discussed here, as well as other data indicating that a complex network of cytokines is likely to have a role in the pathogenesis of MS.

The role of Th1 and Th17 cells in MS. In a small clinical trial in the late 1980s, administration of recombinant IFN- $\gamma$ to individuals with MS, in the hope that it would ameliorate disease, was actually found to induce clinical relapse (18). Many contend these days that Th17 cells are the master mediators of tissue damage in autoimmune conditions such as MS (5). Nonetheless, it is hard to argue with the proposition that Th1 cells are critical in the pathogenesis of relapse in individuals with MS, given that IFN- $\gamma$ is the signature cytokine for Th1 cells and that it worsened disease (18).

Somewhat counter to our understanding of the role of IFN- $\gamma$ from this experience in MS is the observation that administration of IFN- $\gamma$ has the opposite effect in EAE (19). As a result of data from EAE, a role for Th1 cells in MS began to be discounted by many, especially by those who learn most of their lessons about immunology from animal studies, particularly with rodents (4). Specifically, clinical paralysis in various EAE models of MS is ameliorated with administration of IFN- $\gamma$, whereas disease is worse in mice lacking IFN- $\gamma$ and in mice administered blocking antibodies specific for IFN- $\gamma(19,20)$. However, those immunologists who have currently relegated Th 1 cells to a smaller role than Th17 cells in the pathogenesis of autoimmunity in general, especially autoimmune demyelinating disease, must not ignore the fact that IFN- $\gamma$ (the signature cytokine of Th1 cells) worsened the condition of individuals with MS $(4,18)$, and I suggest that it might follow that Th1 cytokines have a critical role in the pathogenesis of MS, while the role of Th17 cytokines is awaiting explication.

In 2001 and 2002, the transcriptional profile of MS lesions at various stages of the disease was described (21-23). In these studies, the presence of transcripts for novel regulators of Th1 cytokines was demonstrated. Osteopontin was one such novel regulator, and although it is a molecule that has never qualified for the designation of cytokine, it greatly influences Th1 and Th17 cytokine production $(4,21)$. Further analysis indicated that osteopontin expression at the protein level was widespread in the brain of individuals with MS $(21,23)$. The transcriptional signature for cytokines in MS lesions also included hallmarks of both Th1 and Th17 cytokine production (22). Writing in 2002, my colleagues and I emphasized that MS lesions contained increased levels of transcripts for genes encoding inflammatory cytokines, in particular IL-6, IL-17, and IFN- $\gamma$, and proteins involved in pathways downstream of these cytokines, as determined by microarray analysis (22). Recent work, has demonstrated the presence of IL-17 in MS lesions (24). Further, the receptors for IL-17 and IL-22 are present on endothelial cells at the BBB (24), and Prat and colleagues have shown that cells of the Th17 lineage can migrate across the $\mathrm{BBB}$, releasing granzyme $\mathrm{B}$, which leads to neuronal death (25). Th17 cells were demonstrated to be among the perivascular lymphocytes, and IL17 mRNA was found to be expressed by astrocytes and oligodendrocytes at the site of active disease in MS lesions (24). So, it seems that the Th17 pathway is likely to be present in the brain of individuals with MS, although its pathophysiologic significance awaits experimental determination. In various EAE models of MS, it is known that Th1 and Th17 cells are independently capable of inducing paralysis and inflammation (26). The pathology in EAE lesions induced by Th1 cells more closely resembles MS than the pathology induced by Th17 cells $(5,26)$. Thus, in MS itself, it remains an open question whether Th17 cells have an actual pathologic role, given that we know that the Th1 cytokine IFN- $\gamma$ is fully capable of inducing worsening of MS $(4,18)$.

IFN- $\beta$ as a treatment for individuals with MS. The three IFN- $\beta$ preparations sold for the treatment of individuals with relapsing-remitting MS are the most widely used class of drugs for this illness. They work in about half of patients, reducing relapse rates by about one-third (27), but there are no known ways to predict who will benefit, despite widespread efforts in this regard, including the application of systems biology and genomics to the question (28). Several known effects of IFN- $\beta$ fit nicely into a realistic mechanism of action, but a combination of many effects is likely to contribute to its clinical success. First, expression of HLA class II (which is needed to activate pathogenic $\mathrm{CD}^{+} \mathrm{T}$ cells) is elevated in the brain of individuals with MS, and IFN- $\beta$ downregulates expression of HLA class II $(27,29)$. Second, although IFN- $\beta$ first upregulates expression of IFN- $\gamma$, after a few weeks of treatment, it eventually downregulates expression of IFN- $\gamma$ (27). Last, IFN- $\beta$ downregulates expression of MMPs such as MMP-2 and MMP-9, both of which play a role in increasing the permeability of the $\operatorname{BBB}(30,31)$. Work from Benveniste's laboratory showed that administration of IFN- $\beta$ reduces steady-state levels of MMP2 mRNA via suppression of $M M P 9$ promoter activity (32). This effect was dependent on the transcription factor STAT $1 \alpha$ (32). Consistent with the hypothesis that IFN- $\beta$ downregulation of MMPs contributes to its beneficial effects in individuals with MS is the observation that blockade of MMPs reverses EAE (33).

Currently, there are no published data indicating that therapeutic administration of IFN- $\beta$ has an effect on the Th17 pathway, although there is evidence that it downregulates the Th1 pathway, as it downregulates expression of the quintessential Th1 cytokine, IFN- $\gamma(27)$. Further, some recent studies are helping to clarify how IFN- $\beta$ modulates proinflammatory cytokines (34). Data from Cantor and colleagues indicate that IFN- $\beta$ negatively regulates Th17 cells in EAE via a mechanism in which osteopontin plays a key role (34). They show that engagement of the type I IFN receptor (IFNAR) on DCs inhibits IL-17 production by T cells via inhibition of intracellular osteopontin (34). Earlier work had shown that inhibition of osteopontin can lower levels of Th1 cytokines as well (21). In addition, administration of osteopontin itself can trigger disease relapses in EAE (35), and expression of osteopontin is elevated in the plasma of patients with MS around the time of relapse $(36,37)$. So, the clinical control of relapses by IFN- $\beta$ may be mediated, in part, via negative regulation of proinflammatory cytokines following inhibition of osteopontin downstream of IFN- $\beta$ triggering of IFNAR.

Although there has been no experimental determination of a role for Th17 cells in the pathogenesis of MS, several studies analyzing whether the beneficial effects of IFN- $\beta$ treatment in individuals with MS occur via modulation of the Th17 pathway have recently been commenced, and data generated in EAE models of MS provide some support for this idea. Specifically, mice with defects in TRIF-dependent type 1 IFN production develop more severe EAE than wild-type mice, in part because signaling through IFNAR was essential for the negative regulation of Th17 cells (38).

Another remarkable association between IFN- $\beta$ and MS comes from a recent proteomic analysis of MS lesions at different stages of disease (39). Active and chronic active MS lesions expressed abundant amounts of tissue factor (TF), a critical protein in the coagulation cascade (38). TF catalyzes the formation of thrombin from prothrombin, and inhibition of this TF-regulated thrombin 


\section{Table 1}

Comparison of the role of cytokines and the differences in inflammation in $A D$ and $M S$

\begin{tabular}{lll}
\hline & MS & AD \\
Characteristic of inflammation & & - \\
Perivascular lymphocyte cuffs & + & - \\
Immunoglobulin in plaques & + & - \\
T cells in plaques & + & - \\
B cells in plaques & + & + \\
Complement in plaques & + & + \\
Cytokine & & + \\
$\quad$ IL-1 $\beta$ & + & + \\
TNF- $\alpha$ & + & + \\
TGF- $\beta$ & + & \\
\hline
\end{tabular}

+, present; -, absent.

pathway ameliorated ongoing EAE, reducing the levels of Th1 and Th17 cytokines as well as reducing phosphorylation of NF-KB (39). Of interest, TF is a member of the class 2 cytokine receptor family, which also includes the receptors for IFN- $\alpha$, IFN- $\gamma$, and IL-10 (40).

Another element of the coagulation cascade, protein $\mathrm{C}$ inhibitor, was expressed at high levels in chronic active MS lesions (39). Together with the observation that levels of protein $\mathrm{C}$ in the serum of patients with MS are lower than those in the serum of healthy individuals $(39,41)$, this suggests that the protein $C$ pathway is suppressed in individuals with MS. Further, the ability of recombinant activated protein $\mathrm{C}$ to ameliorate $\mathrm{EAE}$, an effect associated with decreased expression of Th 1 and Th17 cytokines in astrocytes and immune cells, relied on its function as an anticoagulant and its ability to initiate a signaling cascade involving NF- $\mathrm{KB}$ (39). The presence of fibrin and elements of the coagulation cascade (TF and protein C inhibitor) in MS lesions might therefore have profound effects on the inflammatory response and indicate that they participate in autoimmunity (39).

Blockade of TNF- $\alpha$ : beneficial in RA, damaging in MS. Another cytokine that has been considered as a therapy for MS is TNF- $\alpha$. Strikingly, however, drugs that block TNF- $\alpha$, which are highly effective in the treatment of individuals with RA, lead to worsening of MS, and warnings about this have been posted by regulatory authorities in both the United States and Europe (42-45). This is perhaps not surprising, however, as in the context of the CNS, TNF- $\alpha$ has neuroprotective properties (46). Conversely, while treatment with IFN- $\beta$ can be beneficial to individuals with MS, it has no effect on individuals with RA (47). This raises the obvious observation that the pathology in disease-affected joints in individuals with RA is not identical to the pathology in the CNS of individuals with MS.

In a healthy immune system, IFN- $\beta$, IFN- $\gamma$, and TNF- $\alpha$ work cooperatively to protect the host from pathogens $(48,49)$. It is therefore likely that therapies involving these cytokines, such as IFN- $\beta$ in MS and blockade of TNF- $\alpha$ in RA, also affect the expression and function of the other cytokines in this cooperative network. Indeed, understanding how therapeutic administration of IFN- $\beta$ might modulate expression of TNF- $\alpha$ would be valuable in developing strategies to improve the efficacy and to reduce side effects of this therapy. Despite this knowledge of the effect of TNF- $\alpha$ blockade in MS, and the puzzling absence of an effect of IFN- $\beta$ in RA, the extensive interplay between these cytokines during IFN $-\beta$ treatment in MS has never been carefully elucidated in models of MS or in MS itself. Therefore, a good explanation awaits us as to why IFN- $\beta$ is ineffective in RA, where TNF- $\alpha$ is pathogenic, whereas IFN- $\beta$ is effective in MS, where TNF- $\alpha$ might be, in balance, neuroprotective. Work in elucidating the protective effects of IFN- $\beta$ and TNF- $\alpha$ in EAE models has never established the possible connection between these two major cytokines $(50,51)$. However, recent work in my laboratory indicates that, surprisingly, IFN- $\beta$ increases expression of TNF- $\alpha$ (unpublished observations).

The role of TGF- $\beta$ in MS. The pathophysiologic roles of TGF- $\beta$ are critical in MS and, as discussed below, in AD. The role of TGF- $\beta$ is perhaps better elucidated in MS and in its animal model EAE than it is in AD. In MS, the transcription factor Smad6, which is required for both expression of TGFB and regulation of TGF- $\beta$ signaling, has been found at elevated levels in brains from individuals with MS, including those with active and those with chronic disease (22). Transcripts for TGFB3 have also been found to be upregulated in chronic MS lesions (22), whereas transcripts encoding other isoforms of TGF- $\beta$, including TGF- $\beta 1$ and TFG- $\beta 2$, have been found in both active and chronic MS lesions (52).

In MS, TGF- $\beta$ may be involved in suppression of inflammation late in the chronic stages of disease. However, in the relapsing-remitting early phase of MS, TGF- $\beta 1$ activation is associated with heightened inflammation as it is in EAE models of the disease (53). Further, pharmacologic blockade of TGF- $\beta 1$ can inhibit EAE, reducing expression of IL- 6 and subsequent brain inflammation (53). It is noteworthy that in the later stages of MS, levels of both TGF- $\beta 1$ and IL- 17 are elevated (22). This observation can be united with the finding that IL-6 and Smad6 are elevated throughout the course of MS (20). Since it is currently thought that IL-17 is likely to have a proinflammatory role in MS (although this awaits definitive experimental proof), and that IL-17 production is dependent on optimal concentrations of TGF- $\beta$ (54), TGF- $\beta$ expression in chronic MS along with IL- 6 may drive a Th17 response, initiating chronic tissue damage (3-5). This would be consistent with results in EAE, where mice lacking IL-17 display paralysis acutely but later have a much attenuated course of disease (55).

\section{AD}

Introduction to $A D$ : an inflammatory condition? The most common form of dementia, $\mathrm{AD}$ affects $0.6 \%$ of persons aged $65-69$ years, $1.0 \%$ of persons aged $70-74$ years, $2.0 \%$ of persons aged $75-79$ years, $3.3 \%$ of persons aged $80-84$ years, and $8.4 \%$ of persons aged 85 years and older (56). It is characterized by progressive memory deficits, cognitive impairment, and personality changes. These symptoms are a result of neuronal death, especially in the limbic and association cortices, which have roles in memory and navigation. Neuronal death follows a period of progressive synaptic dysfunction caused by the senile plaques (deposits of amyloid $\beta$ [A $\beta$ ] peptide associated with degenerative neural structures and nonneuronal cells such as microglial cells) and neurofibrillary tangles (aggregations of a hyperphosphorylated form of the microtubuleassociated protein tau) that are the characteristic pathologies of the disease. The relative contributions of $A \beta$ and tau to the process of neuronal death is the subject of intense debate in the field.

Although $\mathrm{AD}$ is considered a neurodegenerative disease, it is clear that it has many attributes of an inflammatory response, with proinflammatory cytokines (notably IL-1 $\alpha$ and IL-1 $\beta$ ) being found throughout the brain of individuals with $\mathrm{AD}$ when analyzed at autopsy (57). The inflammatory response in $\mathrm{AD}$ has been reviewed in great detail recently (58). 
While MS and AD are both pathologic inflammatory conditions of the CNS, perivascular inflammatory cuffs containing lymphocytes and macrophages that fill the CNS of individuals with MS are conspicuously absent in AD. Indeed, in MS, one finds all the cellular components necessary for the promulgation of an adaptive immune response within the confines of the CNS. In addition, in MS, there are also copious quantities of Ig synthesized within the CNS, while in AD this is not the case. Although evidence for cellular and humoral immunity is not present in the brain of individuals with $\mathrm{AD}$, cytokines including TNF- $\alpha$ and IL-1 as well as other components of the immune response, such as members of the complement cascade, are present (58). A comparison of the role of cytokines and the differences in inflammation in $\mathrm{AD}$ and $\mathrm{MS}$ is shown in Table 1. Small clinical trials in AD have been performed with etanercept, which is a fusion protein comprising human soluble TNFR linked to the Fc component of human IgG1 (59). Although some benefit was observed, firm conclusions cannot be drawn at present from these small trials.

The role of TGF- $\beta$ in $A D$. There are certain common aspects of TGF- $\beta$ signaling involved in the pathogenesis of MS and AD. Of interest, transcription of $S M A D 3$, a transcription factor that promotes TGFB expression, was found to be decreased in the brain of individuals with $\operatorname{AD}(58,60)$. As in MS, TGF- $\beta$ might have dual proinflammatory and antiinflammatory roles, although the precise effects of TGF- $\beta$ in AD are not well understood. Indeed, the pathophysiologic roles of TGF- $\beta$ are perhaps much better elucidated in MS and in its animal model EAE, as described in the previous section. However, characterization of the roles of TGF- $\beta$ in $\mathrm{AD}$ has been addressed in experimental models of the disease.

In mice engineered to overexpress amyloid precursor protein (APP) in brain, overexpression of TGF- $\beta$ reduced plaque burden (61). Activation of TGF- $\beta$ in microglial cells might lead therefore to increased degradation of $A \beta$ (61). Somewhat paradoxically, blockade of TGF- $\beta$ signals via Smad $2 / 3$ signaling (using both genetic and pharmacological approaches), instead of reducing $A \beta$ clearance, actually enhanced such clearance and reduced amyloid plaque burden in a transgenic model of AD (62). In this approach, there was increased macrophage infiltration of the brain, reduced proinflammatory TNF- $\alpha$ expression, and increased antiinflammatory IL-10 expression in homogenates from affected brain where TGF- $\beta$ signals were blocked (62). In a separate study, blocking signals through the receptor for TGF- $\beta$ using a pharmacological agent reduced inflammation in EAE, with diminished proinflammatory IL-6 expression (53). Inhibition of TGF- $\beta$ signaling is thus associated with reduced inflammation in the brain in animal models of both AD and MS. Thus, inhibition of TGF- $\beta$ signaling might be a viable approach for the treatment of both $\mathrm{AD}$ and MS.

\section{Summary}

Cytokines are key participants in the febrile response, MS, and AD. Their roles are nuanced and highly regulated. Sometimes the roles of cytokines are diametrically opposite to their function in other diseases, as we have seen for TNF- $\alpha$ in MS and RA. At other times, there is overlap in their function, as we have seen for TGF- $\beta$ in animal models of MS and AD. So far there has not been a cytokine in brain disorders whose role is as paramount as that of TNF- $\alpha$ in RA (41). Nevertheless, the role of IL-1 is critical in fever (8-10), while IFN- $\beta$ has achieved the status of being the most popular drug for amelioration of exacerbations of MS $(27,29)$. The role of cytokines in $\mathrm{AD}$ is much less clear at this point, yet common benefits are seen in impeding TGF- $\beta$ signaling in animal models of AD and MS $(53,62)$, and blockade of the receptor for TGF- $\beta$ may yet be found to have therapeutic benefit in MS and AD.

Going forward, a great deal needs to be learned about the interplay of cytokines in complex diseases such as MS and AD. We need to understand how molecules such as osteopontin can regulate entire pathways such as Th1 and Th17 by interacting with cytokines and their receptors. Whole systems such as the coagulation cascade and the complement cascade also have remarkable intersections with cytokines, and as greater understanding of these interactions is gained, we will have to use additional tools, for example systems biology, to address the complexity of the problem. Or we might just get lucky and find the pivotal molecules in MS and $\mathrm{AD}$, whose manipulation will achieve the spectacular success that we have seen with TNF- $\alpha$ blockade in RA.

Address correspondence to: Lawrence Steinman, Department of Neurology and Neurological Sciences, Interdepartmental Program in Immunology, Stanford University, Stanford, California 94305, USA. Phone: (650) 725-6401; Fax: (650) 725-0627; E-mail: steinman@stanford.edu.
1. Steinman, L. 2004. Elaborate interactions between the immune and nervous systems. Nat. Immunol. 5:575-581.

2. Kastelein, R.A., Hunter, C.A., and Cua, D.J. 2007. Discovery and biology of IL-23 and IL-27: related but functionally distinct regulators of inflammation. Annu. Rev. Immunol. 25:221-242.

3. Bettelli, E., Oukka, M., and Kuchroo, V.K. 2007. $\mathrm{T}(\mathrm{H})-17$ cells in the circle of immunity and autoimmunity. Nat. Immunol. 8:345-350.

4. Steinman, L. 2007. A brief history of TH17, the first major revision in the TH1/TH2 hypothesis of T cellmediated tissue damage. Nat. Med. 13:139-145.

5. Steinman, L. 2008. A rush to judgment on Th17. J. Exp. Med. 205:1517-1522.

6. Auron, P.E., et al. 1984. Nucleotide sequence of human monocyte interleukin 1 precursor cDNA. Proc. Natl. Acad. Sci. U. S. A. 81:7907-7911.

7. Lomedico, P.T., et al. 1984. Cloning and expression of murine interleukin-1 cDNA in E. coli. Nature. 312:458-462.

8. Dinarello, C.A. 1984. Interleukin-1. Rev. Infect. Dis. 6:51-95.

9. Dinarello, C.A., and Wolff, S.M. 1982. Molecular basis of fever in humans. Am. J. Med. 72:799-819.

10. Dinarello, C.A. 2004. Infection, fever, and exogenous and endogenous pyrogens: some concepts have changed. J. Endotoxin Res. 10:201-222.

11. Sanchez-Alavez, M., Tabarean, I.V., Behrens, M.M., and Bartfai, T. 2006. Ceramide mediates the rapid phase of febrile response to IL-1beta. Proc. Natl. Acad. Sci. U. S. A. 103:2904-2908.

12. Quan, N., and Banks, W.A. 2007. Brain-immune communications pathways. Brain Behav. Immun. 21:727-735.

13. Blatteis, C.M. 2007. The onset of fever: new insights into its mechanism. Prog. Brain Res. 162:3-14.

14. Schieferdecker, H.L., Rothermel, E., Timmermann, A., Gotze, O., and Jungermann, K. 1997. Anaphylatoxin C5a receptor mRNA is strongly expressed in Kupffer and stellate cells and weakly in sinusoidal endothelial cells but not in hepatocytes of normal rat liver. FEBS Lett. 406:305-308.

15. Perlik,V., Li, Z., Goorha, S., Ballou, I.R., and Blatteis, C.M. 2005. LPS-activated complement, not LPS per se, triggers the early release of PGE2 by Kupffer cells. Am. J. Physiol. Regul. Integr. Comp. Physiol. 289:R332-R339.
16. Negussie, Y., et al. 1992. Detection of plasma tumor necrosis factor, interleukins 6 and 8 during the Jarisch-Herxheimer reaction of relapsing fever. J. Exp. Med. 175:1207-1212.

17. Fekade, D., et al. 1996. Prevention of Jarisch-Herxheimer reactions by treatment with antibodies against tumor necrosis factor-alpha. N. Engl. J. Med. 335:311-315.

18. Panitch, H.S., Hirsch, R.L., Schindler, J., and Johnson, K.P. 1987. Treatment of multiple sclerosis with gamma interferon: exacerbations associated with activation of the immune system. Neurology. 27:1097-1102.

19. Billiau, A., et al. 1998. Enhancement of experimental allergic encephalomyelitis in mice by antibodies against IFN-gamma. J. Immunol. 140:1506-1510.

20. Krakowski, M., and Owens, T. 1996. Interferongamma confers resistance to experimental allergic encephalomyelitis. Eur. J. Immunol. 26:1641-1646.

21. Chabas, D., etal. 2001. The influence of the pro-inflammatory cytokine, osteopontin, on autoimmune demyelinating disease. Science. 294:1731-1735.

22. Lock, C., et al. 2002. Gene microarray analysis of multiple sclerosis lesions yields new targets vali- 
dated in autoimmune encephalomyelitis. Nat. Med. 8:500-508.

23. Steinman, L., and Zamvil, S. 2003. Transcriptional analysis of targets in multiple sclerosis. Nat. Rev. Immunol. 3:483-493.

24. Tzartos, J.S., et al. 2008. Interleukin-17 production in central nervous system-infiltrating $\mathrm{T}$ cells and glial cells is associated with active disease in multiple sclerosis. Am. J. Pathol. 172:146-155.

25. Kebir, H., et al. 2007. Human TH17 lymphocytes promote blood-brain barrier disruption and central nervous system inflammation. Nat. Med. 13:1173-1175

26. Kroenke, M.A., Carlson, T.J., Adnjelkovic, A.V., and Segal, B.M. 2008. IL-12- and IL-23-modulated T cells induce distinct types of EAE based on histology, CNS chemokine profile, and response to immunomodulatory intervention. J. Exp. Med. 205:1535-1541.

27. Arnason, B.G.W. 1999. Immunologic therapy of multiple sclerosis. Ann. Rev. Med. 50:291-302.

28. Byun, E., et al. 2008. Genome-wide pharmacogenomic analysis of the response to interferon beta therapy in multiple sclerosis. Arch. Neurol. 65:337-344.

29. Steinman, L. 2001. Multiple sclerosis: a two-stage disease. Nat. Immunol. 2:762-765.

30. Stüve, O., et al. 1996. Interferon beta-1b decreases the migration of Tlymphocytes in vitro: effects on matrix metalloproteinase-9. Ann. Neurol. 40:853-863.

31. Uhm, J.H., et al. 1999. Migratory behavior of lymphocytes isolated from multiple sclerosis patients: effects of interferon beta-1b therapy. Ann. Neurol. 46:319-324.

32. Ma. Z., Qin, H., and Benveniste, E.N. 2001. Transcriptional suppression of matrix metalloproteinase-9 gene expression by IFN-gamma and IFN-beta: critical role of STAT-1alpha. J. Immunol. 167:5150-5159.

33. Gijbels, K., Galardy, R., and Steinman, L. 1994. Reversal of EAE with a hydroxymate inhibitor of matrix metalloproteases. J. Clin. Invest. 94:2177-2182.

34. Shinohara, M.L., Kim, J.-H., Garcia, V.A., and Cantor, H. 2008. Engagement of the type I interferon receptor on dendritic cells inhibits T helper 17 cell development: role of intracellular osteopontin. Immunity. 29:68-78.

35. Hur, E., et al. 2007. Osteopontin-induced relapse and progression of autoimmune brain disease through enhanced survival of activated T cells. Nat. Immunol. 8:74-83.

36. Vogt, M.H., Lopatinskaya, L., Smits, M., Polman, C.H., and Nagelkerken, L. 2003. Elevated osteopontin levels in active relapsing-remitting multiple sclerosis. Ann. Neurol. 53:819-822.

37. Vogt, M.H., et al. 2004. Osteopontin levels and increased disease activity in relapsing-remit- ting multiple sclerosis patients. J. Neuroimmunol. 155:155-160.

38. Guo, B., Chang, E.Y., and Cheng, G. 2008. The type I IFN induction pathway constrains Th17-mediated autoimmune inflammation in mice. J. Clin. Invest. 118:1680-1690

39. Han, M.H., et al. 2008. Proteomic analysis of active multiple sclerosis lesions reveals therapeutic targets. Nature. 451:1076-1081.

40. Harlos, K., et al. 1994. Crystal structure of the extracellular region of human tissue factor. Nature. 370:662-666.

41. Kirichuk, V.F., and Streknev, A.G. 2003. The role of hemostasis system in the pathogenesis and course of multiple sclerosis [In Russian]. Zh. Nevrol. Psikhiatr. Im. S. S. Korsakova. 103(Special Issue 2):34-38.

42. U.S. Food and Drug Administration. 2001. Safety update on TNF- $\alpha$ antagonists: infliximab and etanercept.http://www.fda.gov/OHRMS/DOCKETS/ac/01/ briefing/3779b2_01_cber_safety\%20_revision2.pdf.

43. Feldmann, M., and Maini, R.N. 2003. Lasker Clinical Medical Research Award. TNF defined as a therapeutic target for rheumatoid arthritis and other autoimmune diseases. Nat. Med. 9:1245-1250.

44. van Oosten, B.W., et al. 1996. Increased MRI activity and immune activation in two multiple sclerosis patients treated with the monoclonal antitumor necrosis factor antibody cA2. Neurology. 47:1531-1534.

45. [No authors listed.] 1999. TNF neutralization in MS: results of a randomized, placebo-controlled multicenter study. The Lenercept Multiple Sclerosis Study Group and The University of British Columbia MS/MRI Analysis Group. Neurology. 53:457-465.

46. Turrin, N.P., and Rivest, S. 2006. Tumor necrosis factor alpha but not interleukin 1 beta mediates neuroprotection in response to acute nitric oxide excitotoxicity. J. Neurosci. 26:143-151.

47. van Holten, J., et al. 2005. A multicentre, randomised, double blind, placebo controlled phase II study of subcutaneous interferon beta-1a in the treatment of patients with active rheumatoid arthritis. Ann. Rheum. Dis. 64:64-69.

48. Nguyen, K.B., et al. 2002. Critical role for STAT4 activation by type 1 interferons in the interferon-gamma response to viral infection. Science. 297:2063-2066.

49. Wong, L.H., Hatzinisiriou, I., Devenish, R.J., and Ralph, S.J. 1998. IFN-gamma priming up-regulates IFN-stimulated gene factor 3 (ISGF3) components, augmenting responsiveness of IFN-resistant melanoma cells to type I IFNs. J. Immunol. 160:5475-5484.

50. Axtell, R., and Steinman, L. 2008. Type 1 interferons cool the inflamed brain. Immunity. 28:600-602.

51. Prinz, M., et al. 2008. Distinct and nonredundant in vivo functions of IFNAR on myeloid cells limit autoimmunity in the central nervous system. Immunity. 28:675-686.

52. De Groot, C.J., Montagne, L., Barten, A.D., Sminia, P., and Van Der Valk, P. 1999. Expression of transforming growth factor (TGF)-beta1, -beta2, and -beta3 isoforms and TGF-beta type I and type II receptors in multiple sclerosis lesions and human adult astrocyte cultures. J. Neuropathol. Exp. Neurol. 58:174-187.

53. Luo, J., et al. 2007. Glia-dependent TGF-beta signaling, independent of the TH17 pathway, is critical for initiation of autoimmune encephalomyelitis. J. Clin. Invest. 117:3306-3315.

54. Manel, N., Unutmaz, D., and Littman, D.R. 2008. The differentiation of human Th17 cells requires transforming growth factor-beta and induction of the nuclear receptor RORgammat. Nat. Immunol. 9:641-649.

55. Komiyama, Y., et al. 2006. IL-17 plays an important role in the development of experimental autoimmune encephalomyelitis. J. Immunol. 177:566-573.

56. Hebert, L.E., et al. 1995. Age-specific incidence of Alzheimer's disease in a community population. JAMA. 273:1354-1359.

57. Griffin, W.S., et al. 1989. Brain interleukin 1 and S-100 immunoreactivity are elevated in Down syndrome and Alzheimer disease. Proc. Natl. Acad. Sci. U. S. A. 86:7611-7615.

58. Wyss-Coray, T. 2006. Inflammation in Alzheimer disease: driving force, bystander or beneficial response? Nat. Med. 12:1005-1015.

59. Tobinick, E.L., and Gross, H. 2008. Rapid improvement in verbal fluency and aphasia following perispinal etanercept in Alzheimer's disease. BMC Neurol. 8:27.

60. Colangelo, V., et al. 2002. Gene expression profiling of 12633 genes in Alzheimer hippocampal CA1: transcription and neurotrophic factor down-regulation and up-regulation of apoptotic and pro-inflammatory signaling. J. Neurosci. Res. 70:462-473.

61. Wyss-Coray, T., et al. 2001. TGF-beta1 promotes microglial amyloid-beta clearance and reduces plaque burden in transgenic mice. Nat. Med. 7:612-618.

62. Town, T., et al. 2008. Blocking TGF-beta-Smad2/3 innate immune signaling mitigates Alzheimer-like pathology. Nat. Med. 14:681-687.

63. Feldmann, M., and Steinman, L. 2005. Design of effective immunotherapy for human autoimmunity. Nature. 435:612-619.

64. Steinman, L. 2005. Blocking adhesion molecules as therapy for multiple sclerosis: natalizumab. Nat. Rev. Drug Discov. 4:510-518. 